\title{
Serum lipidomic profiling as a useful tool for screening potential biomarkers of hepatitis B-related hepatocellular carcinoma by ultraperformance liquid chromatography-mass spectrometry
}

\author{
Ana Maria Passos-Castilho ${ }^{1 *}$ D, Valdemir Melechco Carvalho², Karina Helena Morais Cardozo ${ }^{2}$, Luciana Kikuchi ${ }^{3}$, \\ Aline Lopes Chagas ${ }^{3}$, Michele Soares Gomes-Gouvêa ${ }^{3}$, Fernanda Malta ${ }^{3}$, Ana Catharina de Seixas-Santos Nastri ${ }^{4}$, \\ João Renato Rebello Pinho ${ }^{3}$, Flair José Carrilho ${ }^{3}$ and Celso Francisco Hernandes Granato ${ }^{1,2}$
}

\begin{abstract}
Background: Chronic hepatitis B (CHB) virus infection is a major cause of hepatocellular carcinoma (HCC), as late diagnosis is the main factor for the poor survival of patients. There is an urgent need for accurate biomarkers for early diagnosis of HCC. The aim of the study was to explore the serum lipidome profiles of hepatitis B-related HCC to identify potential diagnostic biomarkers.

Methods: An ultraperformance liquid chromatography mass spectrometry (UPLC-MS) lipidomic method was used to characterize serum profiles from HCC ( $n=32)$, liver cirrhosis (LC) $(n=30), \mathrm{CHB}(n=25)$, and healthy subjects $(n=34)$. Patients were diagnosed by clinical laboratory and imaging evidence and all presented with CHB while healthy controls had normal liver function and no infectious diseases.

Results: The UPLC-MS-based serum lipidomic profile provided more accurate diagnosis for LC patients than conventional alpha-fetoprotein (AFP) detection. HCC patients were discriminated from LC with $78 \%$ sensitivity and $64 \%$ specificity. In comparison, AFP showed sensitivity and specificity of $38 \%$ and $93 \%$, respectively. HCC was differentiated from CHB with $100 \%$ sensitivity and specificity using the UPLC-MS approach. Identified lipids comprised glycerophosphocolines, glycerophosphoserines and glycerophosphoinositols.
\end{abstract}

Conclusions: UPLC-MS lipid profiling proved to be an efficient and convenient tool for diagnosis and screening of HCC in a high-risk population.

Keywords: Biomarker, Hepatocellular carcinoma, Hepatitis B, Lipidomics, UPLC-MS, Diagnosis

\section{Background}

Hepatitis B virus (HBV) infection is one of the main causes of chronic liver disease worldwide. It is estimated that 240 million individuals are chronically infected with HBV [1]. Depending on the presence of co-factors, progression to liver cirrhosis (LC) may occur at a rate of 2 to $10 \%$ per year, whereas hepatocellular carcinoma (HCC)

\footnotetext{
* Correspondence: anampassos@gmail.com

1 Division of Infectious Diseases, Federal University of Sao Paulo, 781 Pedro

de Toledo Street, 15th floor, Sao Paulo, SP 04039032, Brazil

Full list of author information is available at the end of the article
}

() may develop in $2-4 \%$ of patients per year. HBV is estimated to be responsible for $30 \%$ of cirrhosis- and $45 \%$ of HCC-related deaths [2].

In Brazil, HBV accounts for 13-25\% of HCC cases in most geographical regions, reaching $40 \%$ of $\mathrm{HCC}$ cases in the Mid-west [3].

$\mathrm{HCC}$ is a complex and heterogeneous tumor with several genomic alterations and its incidence has been increasing worldwide. It is the sixth most common cancer and the second cause of cancer-related death. When diagnosed at an early stage, surgical options such as 
resection or liver transplantation, or local ablative therapies, can be applied with intent to cure HCC. However, until now, no effective serum or plasma biomarkers have been found for accurate screening or diagnosis of $\mathrm{HCC}[4,5]$.

HCC diagnosis is most commonly performed by ultrasound examination, CT scan and/or magnetic resonance. Histopathology confirmation may also be necessary in some cases. However, there are some limitations related to risk of complications and feasibility of the biopsy due to tumor location. Moreover, the effectiveness of ultrasound for early detection of HCC is highly dependent on the stage of liver fibrosis, the quality of the equipment and the expertise of the operator [4].

Alpha-fetoprotein (AFP) is the most widely used biomarker for HCC. However, its sensitivity is only up to $60 \%$ and elevated AFP levels are also common in LC and chronic liver disease [6]. Thus, there is an urgent need to identify better HCC biomarkers.

The ideal biomarker should be specific and able to discriminate $\mathrm{HCC}$ from regenerative nodules irrespective of the stage of liver disease. Furthermore, the biomarker should be sensitive, allowing detection at an early stage, and should be easily measurable, reproducible, and minimally invasive.

Recently developed mass spectrometry (MS)-based techniques such as lipidomics are promising tools for the discovery and subsequent identification of molecules associated with various diseases. Separation techniques, like ultraperformance liquid chromatography (UPLC), coupled to MS enable the analysis of complex samples such as plasma or serum with very high sensitivity and accuracy $[7,8]$.

Once lipid biomarkers are identified through UPLCMS, they can be later investigated in clinical laboratory routine using simple and accessible colorimetric and/or enzymatic techniques. Nonetheless, studies on lipid profiling and fingerprinting of HCC are still scarce [9-12].

The aim of this study was to assess the serum lipid patterns of HCC by performing UPLC-MS to search for potential biomarkers for diagnosis in $\mathrm{HBV}$ chronic infected patients (HBV-HCC).

\section{Methods}

\section{Study design, sample and data collection}

A total of 87 patients with chronic hepatitis $\mathrm{B}(\mathrm{CHB})$ were enrolled from 2012 to 2014 at the Hospital das Clínicas of the University of Sao Paulo School of Medicine, including 32 patients with HBV-HCC, 30 patients with HBV-LC and 25 patients with $\mathrm{CHB}$. Additionally, 34 eligible blood donors with normal liver function and no infectious diseases were recruited at COLSAN Beneficent Association for Blood Collection to serve as healthy controls. $\mathrm{CHB}$ was diagnosed based on the presence of HBsAg for at least 6 months. LC was diagnosed by histopathology, clinical features and/or elastography and HCC was diagnosed using imaging or histopathology techniques, in accordance with guidelines of the Brazilian Society of Hepatology.

Blood samples were obtained by venipuncture and drained into blood collection tubes. The samples were centrifuged immediately after collection and serum was stored at $-80{ }^{\circ} \mathrm{C}$ until analysis.

Demographic, clinical and laboratory data were collected from medical records. The study was approved by the ethics committee of human research of the Federal University of Sao Paulo and the University of São Paulo School of Medicine (2012/81656 and 2014/569922) and all patients gave written informed consent.

\section{Extraction of lipids}

Lipids were extracted from each sample using a modified Bligh-Dyer protocol [13]. Immediately after thawing, $100 \mu \mathrm{L}$ of serum were dissolved in $850 \mu \mathrm{L}$ of a mixture of water/chloroform/methanol (1:2.5:5, v/v) and vortexed well for $5 \mathrm{~min}$. After vortexing, $250 \mu \mathrm{L}$ of chloroform were added and the tubes were agitated for $15 \mathrm{~min}$ at $700 \mathrm{rpm}$. Then, $200 \mu \mathrm{L}$ of deionized water were added and the tubes were centrifuged at $14,000 \mathrm{rpm}$ for $15 \mathrm{~min}$ at room temperature. Following this protocol a 2-phase system (aqueous top, organic bottom) was achieved. The bottom phase containing lipids was gently recovered using a micropipette, dried, and resuspended in $350 \mu \mathrm{L}$ of acetonitrile/water $(3: 2, \mathrm{v} / \mathrm{v})$. All chemicals were of analytical reagent grade and used as received.

\section{UPLC-MS analysis}

Reversed-phased analysis was performed on a Waters ACQUITY IClass UPLC system equipped with a Waters Acquity CSH C18 $1.7 \mu \mathrm{m}$ x $2.1 \times 100$ mm column coupled to a Waters Synapt-MS hidrid quadrupoletime of flight mass spectrometer operating in the positive ion electrospray mode. A mass scan range of 200 to 1,200 mass-to-charge ratio $(\mathrm{m} / \mathrm{z})$ was set for data acquisition in continuous mode with optimized parameters for ionization and mass transmission. Acetonitrile/ water $(3: 2, \mathrm{v} / \mathrm{v})$ was used as mobile phase A and isopropanol/acetonitrile $(9: 1, \mathrm{v} / \mathrm{v})$ was used as mobile phase $\mathrm{B}$, both with $10 \mathrm{mM}$ ammonium formate and $0.1 \%$ formic acid as additives. The flow rate was set at $600 \mu \mathrm{L} / \mathrm{min}$ and the injection volume was $10 \mu \mathrm{L}$. A binary gradient was optimized as follows: the composition of mobile phase B was changed from $15 \%$ to $30 \%$ in $2 \mathrm{~min}$, then to $48 \%$ in $30 \mathrm{~s}$ and reached $82 \%$ in $8.5 \mathrm{~min}$. Subsequently, it was changed to $99 \%$ in $30 \mathrm{~s}$, held for another $30 \mathrm{~s}$ and then dropped to $15 \%$ in $6 \mathrm{~s}$ prior to being held until a total run time of $15 \mathrm{~min}$. The mass spectrometer was previously calibrated with $0.1 \%$ phosphoric acid in water/acetonitrile $(1: 1, \mathrm{v} / \mathrm{v})$ and a solution of $0.5 \mathrm{ng} / \mu \mathrm{L}$ leucine enkephalin in water/ 
Table 1 Demographic data of the enrolled population of the study by group

\begin{tabular}{llllll}
\hline Characteristics & $\mathrm{HS}$ & $\mathrm{CH}$ & $\mathrm{LC}$ & HCC & $P$ \\
\hline Number & 34 & 25 & 30 & 32 & - \\
Mean age $\pm \mathrm{SD}$ & $42.6 \pm 14.8$ & $37.1 \pm 14.2$ & $56.8 \pm 11.0$ & $59.0 \pm 11.3$ & 0.447 \\
Median age & 42.0 & 35.0 & 56.5 & 57.0 & 0.812 \\
Age range & $21-67$ & $19-63$ & $34-80$ & $38-85$ & - \\
Gender (M/F) & $13 / 21$ & $17 / 8$ & $20 / 10$ & $26 / 6$ & 0.190 \\
\hline
\end{tabular}

HS healthy subjects, $\mathrm{CH}$ chronic hepatitis, $L C$ liver cirrhosis, $H C C$ hepatocellular carcinoma, $S D$ standard deviation, $M$ male, $F$ female

acetonitrile $(1: 1, \mathrm{v} / \mathrm{v})$ with $0.1 \%$ formic acid infused in the reference probe at a flow rate of $5 \mu \mathrm{L} / \mathrm{min}$ was used as lock mass spray at a $30 \mathrm{~s}$ frequency for accurate mass determination. All analyses were acquired using the lock spray and the instrument was recalibrated every 4 run-hours to ensure accuracy and reproducibility. Furthermore, a quality control of pool plasma samples was analyzed after every 10 runs, and 10 peaks well distributed from 200 to $1,200 \mathrm{~m} / z$ were assessed.

\section{Data pretreatment and statistical data analyses}

All data obtained from the UPLC-MS analyses were processed with the Waters Progenesis software (Manchester, UK). This step included mass correction, chromatograms and spectra alignment and peak detection using default parameters. After attribution, the matrix of the features characterized by their $\mathrm{m} / z$ and retention time (RT) was uploaded into the MetaboAnalyst 3.0 (The Metabolomics Innovation Centre, Canada). For normalization, data was mean-centered and divided by the square root of standard deviation of each variable (Pareto scaling).

For multivariate analysis, the unsupervised principal component analysis (PCA) was first utilized in all samples (Additional file 1). Supervised partial least-squareslatent structure discriminate analysis (PLS-DA) was then performed to identify biomarkers that contributed to the clustering. Validation with a permutation test and 100 repetitions was performed to prevent model overfitting. Potential biomarkers that differentiated HCC from LC, $\mathrm{CH}$ and healthy subjects (HS) were selected based on the variable importance in the projection (VIP) values and univariate statistical significance after Mann-Whitney test and fold-change analyses. Receiver operating characteristic (ROC) curves were performed to evaluate the accuracy of the potential biomarkers and the proposed model using the ROCCET (The Metabolomics Innovation Centre, Canada).

Statistical analyses of demographic, clinical and laboratory data of subjects were performed using SPSS version 11.0 (SPSS Inc., Chicago, IL, USA). Descriptive statistics consisted of the characterization of the studied population (demographic, clinical and laboratory characteristics) through the respective percentages or mean/median and standard deviation (SD) for continuous variables. Bivariate analysis consisted of Fisher exact test to compare categorical values. For continuous variables, Student's $t$-test was use to compare means of normally distributed variables, while non-normally distributed variables were subjected to Mann-Whitney $U$ test. Statistical significance level was $P<0.050$. All reported values are 2 tailed.

A tentative identification of the differentiating lipids was performed on the LIPID MAPS and HMDB databases.

\section{Results}

The mean age of patients was 59.0 years old in the HCC group, 56.8 in the LC group, and 37.1 in the CHB group. The mean age of the HS was 42.6 years. In the HCC group $81.3 \%$ of patients were males, while in the LC,

Table 2 Clinical and laboratory data of patients with liver cirrhosis and hepatocellular carcinoma

\begin{tabular}{|c|c|c|c|}
\hline Characteristics & $\mathrm{LC}$ & $\mathrm{HCC}$ & $P$ \\
\hline $\operatorname{AFP}(\mathrm{ng} / \mathrm{mL})$ & $6.2 \pm 15.8$ & $507.8 \pm 1565.9$ & $<0.001^{*}$ \\
\hline AFP $\geq 20 \mathrm{ng} / \mathrm{mL}$ & $2(6.67)$ & $12(37.5)$ & - \\
\hline AFP $\geq 200 \mathrm{ng} / \mathrm{mL}$ & $0(0.0)$ & $6(18.8)$ & - \\
\hline $\operatorname{ALT}(U \mathrm{U} / \mathrm{mL})$ & $35.4 \pm 41.3$ & $39.5 \pm 43.2$ & 0.698 \\
\hline AST (UI/mL) & $37.3 \pm 22.4$ & $45.3 \pm 43.0$ & 0.746 \\
\hline ALP (U/L) & $86.6 \pm 51.6$ & $119.2 \pm 70.1$ & $0.022^{*}$ \\
\hline GGT (U/L) & $64.2 \pm 98.2$ & $108.1 \pm 121.0$ & $0.045^{*}$ \\
\hline Total bilirubin (mg/dL) & $0.7 \pm 0.3$ & $1.1 \pm 1.4$ & 0.481 \\
\hline Albumin (g/dL) & $4.7 \pm 0.4$ & $4.4 \pm 0.8$ & 0.117 \\
\hline Platelets $\left({ }^{*} 1,000 / \mathrm{mm}^{3}\right)$ & $155.8 \pm 89.5$ & $156.0 \pm 86.3$ & 0.910 \\
\hline PT (seconds) & $14.1 \pm 2.1$ & $13.2 \pm 1.7$ & $0.013^{*}$ \\
\hline \multicolumn{4}{|l|}{ Child-Pugh score } \\
\hline A & $30(53.6)$ & $26(46.4)$ & $0.030^{*}$ \\
\hline$B$ or $C$ & $0(0.0)$ & $6(100.0)$ & \\
\hline Total cholesterol (mg/dL) & $179.4 \pm 38.1$ & $172.8 \pm 41.0$ & 0.511 \\
\hline Tumor size (mm) & - & $37.0 \pm 23.0$ & - \\
\hline \multicolumn{4}{|l|}{ BCLC stage } \\
\hline 0 & - & $5(15.6)$ & - \\
\hline$A$ & - & $14(43.8)$ & - \\
\hline B & - & $10(31.2)$ & - \\
\hline C & - & $1(3.1)$ & - \\
\hline$D$ & - & $2(6.3)$ & - \\
\hline
\end{tabular}

Results are presented as number and percentage for categorical variables and as mean value and standard deviation for continuous variables. $L C$ liver cirrhosis, HCC hepatocellular carcinoma, AFP alpha-fetoprotein, ALT alanine aminotransferase, AST aspartate aminotransferase, ALP alkaline phosphatase, GGT gamma glutamyl transpeptidase, $P T$ prothrombin time, BCLC BarcelonaClinic Liver Cancer. *Significant at 0.05 


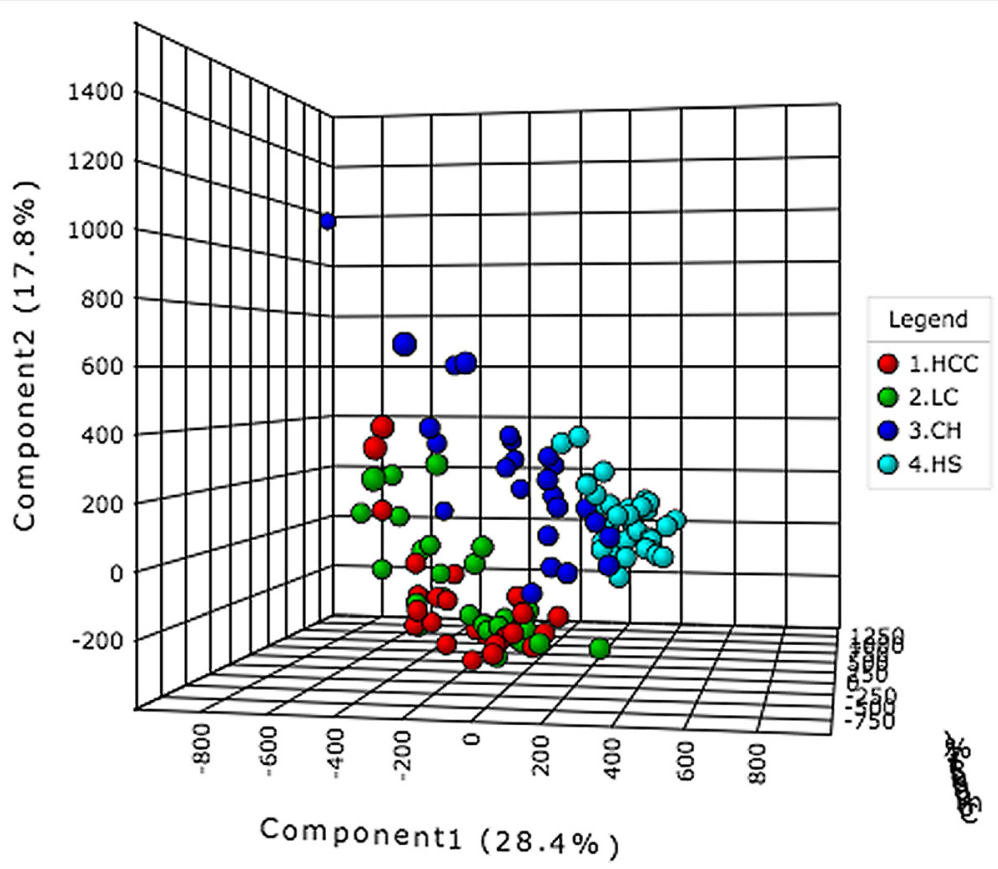

Fig. 1 PLS-DA scores plot based on the UPLC-MS profiling data for the studied groups. Detailed legend: The score plots show the first, second and third latent variables. Each dot in the plot represents a patient according to its group. HCC, hepatocellular carcinoma; LC, liver cirrhosis; CH, chronic hepatitis; HS, healthy subjects

$\mathrm{CH}$ and HS groups they were 66.7, 68.0 and $38.2 \%$, respectively (Table 1 ).

Clinical and laboratory data analyses were performed for the HCC and LC groups (Table 2). In summary, the mean levels of AFP, alkaline phosphatase (ALP), and gamma-glutamyl transpeptidase (GGT) were significantly higher in the HCC group, while prothrombin time (PT) was lower. Nonetheless, the Child-Pugh score distribution was only slightly different between the LC and the HCC groups, which presented 4 and 2 patients with $\mathrm{B}$ and $\mathrm{C}$ scores, respectively. Twenty-eight of the $32 \mathrm{HCC}$ patients (87.5 \%) presented with LC. HCC was classified as BCLC-Barcelona Clínic Liver Cancer staging system very early or early stage in 19 of the 32 cases (59.4\%), intermediate stage in 10 (31.2\%) and advanced or terminal stage in only 3 (9.4\%) cases (Additional file 2.).

A total of 2,698 ions were detected using the UPLCMS method in this study. Figure 1 shows the PLS-DA score plot for the 4 groups evaluated. The separate PLS-DA score plots for inter-group comparisons are shown in Fig. 2.

\section{Hepatocellular carcinoma versus liver cirrhosis}

Four lipids independently predicted HCC from LC with 65.6-84.4\% sensitivity, and 60.0-76.7\% specificity. Figure 3 shows the intensities and ROC curves of the 4 lipids in patients with HCC and LC.
Based on the efficiency of the ROC curves, cutoff values were determined for each ion. The number of "positive" ions in each sample was used to generate a 4peak algorithm with cutoff value of at least 2 "positive" biomarkers, defined by ROC curve analysis and posterior univariate statistical validation (Fig. 4a). The 4-peak algorithm generated distinguished HCC from LC with an accuracy of $71.0 \%$ (95\% CI 58.7-80.1\%), a sensitivity of $78.1 \%$ (95 \% CI 61.2-89.0\%), and a specificity of $63.6 \%$ (95\% CI: 45.4-78.1\%). This algorithm successfully detected 25 of $32 \mathrm{HCC}$ cases when applied to discriminate HCC from LC.

Conversely, AFP detected only 12 of $32 \mathrm{HCC}$ cases from LC when cutoff value was set as $20 \mathrm{ng} / \mathrm{mL}$, showing an accuracy of $64.5 \%$ (95\% CI 52.1-75.3 \%), a sensitivity of $37.5 \%$ (95\% CI $22.9-54.8 \%$ ), and a specificity of $93.3 \%$ (95 \% CI: 78.7-98.2\%). In the range of $200 \mathrm{ng} / \mathrm{mL}$, AFP detected 6 of 32 HCC cases, performing with an accuracy of $58.1 \%$ (95 \% CI 45.7-69.5\%), a sensitivity of $18.8 \%$ (95 \% CI: 8.9-35.3\%), and a specificity of $100 \%$ (95\% CI 88.7-100.0\%) (Table 3).

The accuracy, sensitivity and specificity of HCC detection of the 4-peak algorithm was not compromised when the $6 \mathrm{HCC}$ patients with Child-Pugh scores B and $\mathrm{C}$ were excluded from the analysis. Likewise, the HCC detection rate of the algorithm did not vary significantly when patients were stratified according to the BCLC 


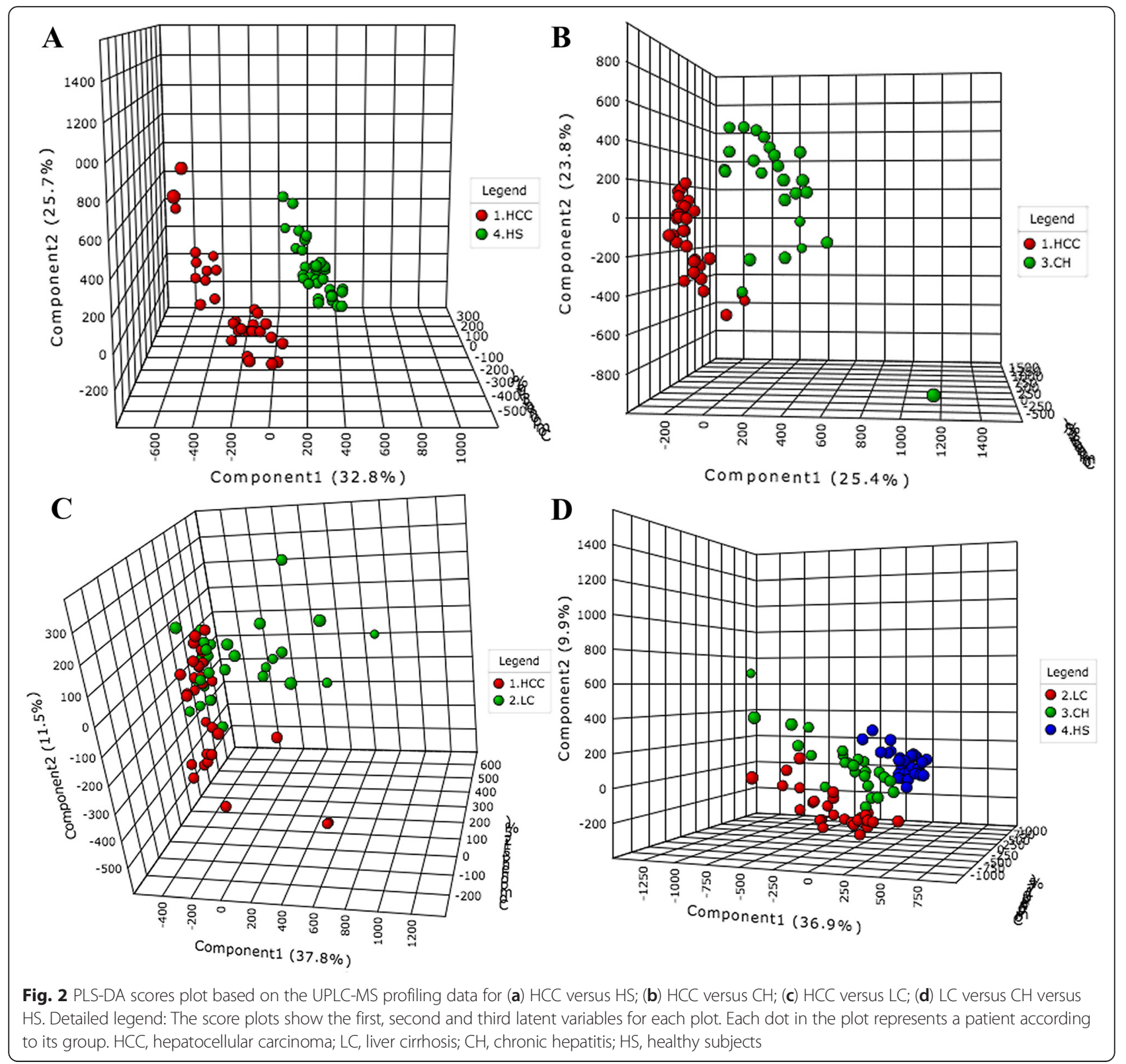

staging system $(P=0.463)$. Very early or early $\mathrm{HCC}$ was detected with a sensitivity of $73.7 \%$ (95 \% CI: $51.2-$ $88.2 \%$ ), and a specificity of $63.3 \%$ (95\% CI: $45.5-78.1 \%$ ).

The combination of the 4-peak UPLC-MS algorithm with AFP in the range of $20 \mathrm{ng} / \mathrm{mL}$ was able to distinguish HCC from LC with an accuracy of $79.0 \%$ (95 \% CI 67.4-87.3\%), a sensitivity of $75 \%$ (95 \% CI 57.9-86.8 \%), and a specificity of $83.3 \%$ (95\% CI: 66.4-92.7\%).

\section{Hepatocellular carcinoma versus chronic hepatitis B}

The 4 peaks independently predicted $\mathrm{HCC}$ from $\mathrm{CHB}$ with $52-90.6 \%$ sensitivity and $68.8-86.7 \%$ specificity. The intensities and ROC curves of the 4 lipids in patients with $\mathrm{HCC}$ and $\mathrm{CHB}$ are shown in Additional file 3. The 4-peak algorithm distinguished $\mathrm{HCC}$ from $\mathrm{CHB}$ with an accuracy of $87.1 \%$ (95\% CI 76.6-93.3\%), a sensitivity of $93.8 \%$ (95\% CI 79.9-98.3\%), and a specificity of 80.0 \% (95 \% CI 62.7-90.5 \%) (Table 3).

As the ion RT 4.26_540.4255 m/z did not perform well in this comparison, we also tested the performance of the model using different combinations of the 4 ions. The best model was a combination of the ion RT 1.30_498.8315 m/z and RT 1.32_497.5731 m/z, which with a cutoff of at least 1 "positive" ion, detected 31 of the 32 HCC cases and distinguished $\mathrm{HCC}$ and $\mathrm{CHB}$ with an accuracy of $88.7 \%$ (95\% CI 78.5-94.4\%), a sensitivity of $96.9 \%$ (95\% CI 84.26-99.5\%), and a specificity of $80.0 \%$ (95 \% CI 62.7-90.5 \%) (Fig. 4b). 

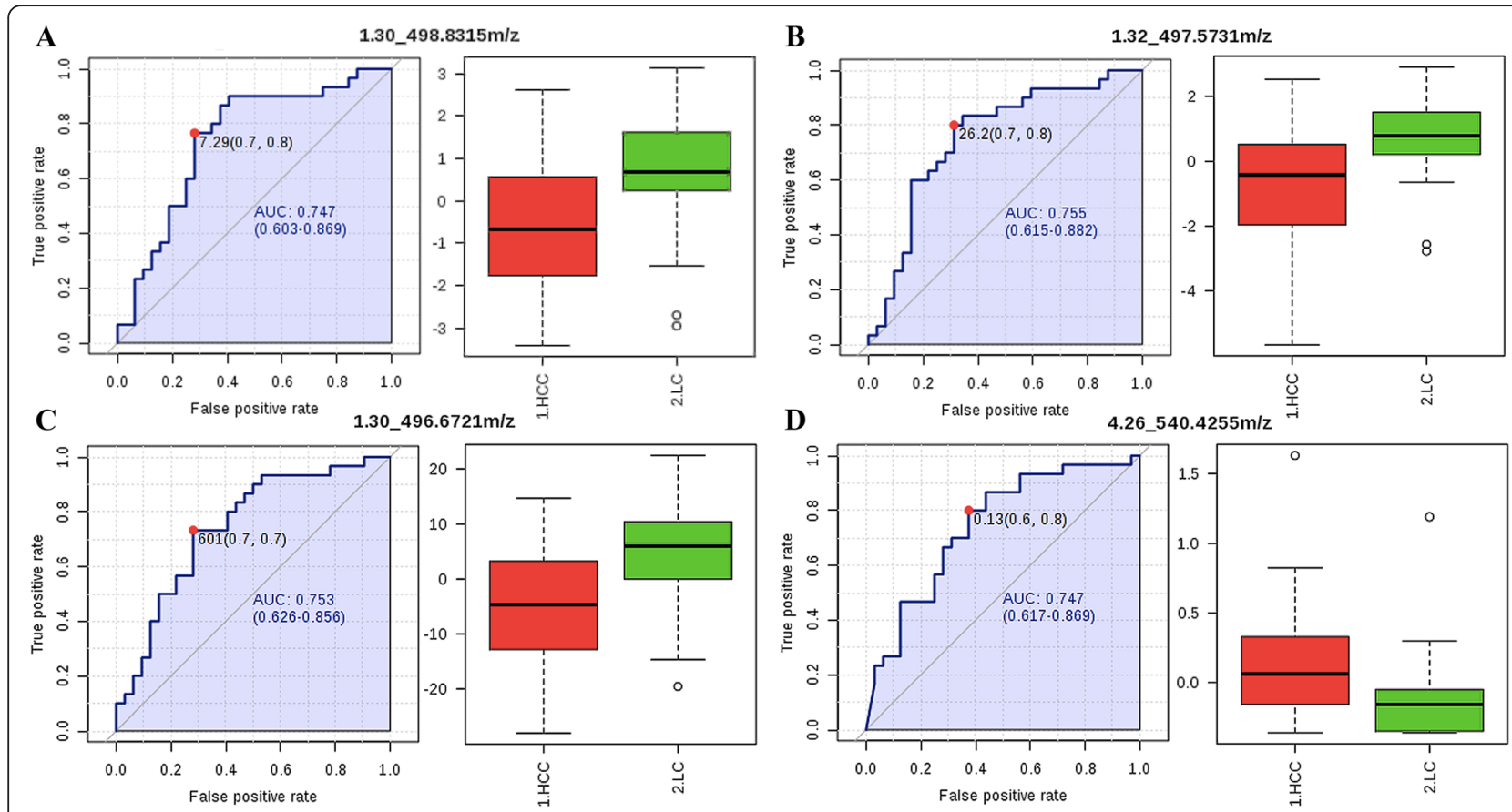

Fig. 3 ROC curves and intensities of the differential ions in the UPLC-MS 4-peak model by RT and $\mathrm{m} / z$. Detailed legend: ROC curves and intensities of the differential ions in the ULC-MS 4-peak model in HCC (red boxes) and LC (green boxes) patients for the ions (a) RT 1.30_498.8315 m/z; (b) RT 1.32_497.5731 m/z; (c) RT 1.30_496.6721 m/z; (d) RT 4.26_540.4255 m/z. AUC, area under the curve; HCC, hepatocellular carcinoma; LC, liver cirrhosis; $\mathrm{CH}$, chronic hepatitis; HS, healthy subjects

We also looked at the whole lipidomic profile of HCC and $\mathrm{CHB}$ and, interestingly, 7 peaks independently predicted HCC from CHB with $100 \%$ sensitivity and specificity (Additional file 4).

\section{Tentative identification of potential biomarkers}

Table 4 shows the main classes and subclasses associated with the differentiating lipids found in this study.

\section{Discussion}

Diagnosis of HCC at an early stage is essential for disease prognosis as it allows the application of curative treatments and improves patient survival.

In the present study, an UPLC-MS-based lipidomic expression signature successfully distinguished HBV-HCC cases from HBV-LC with $78.1 \%$ sensitivity and $63.6 \%$ specificity and provided a more precise diagnostic instrument for cirrhotic patients than conventional non-invasive

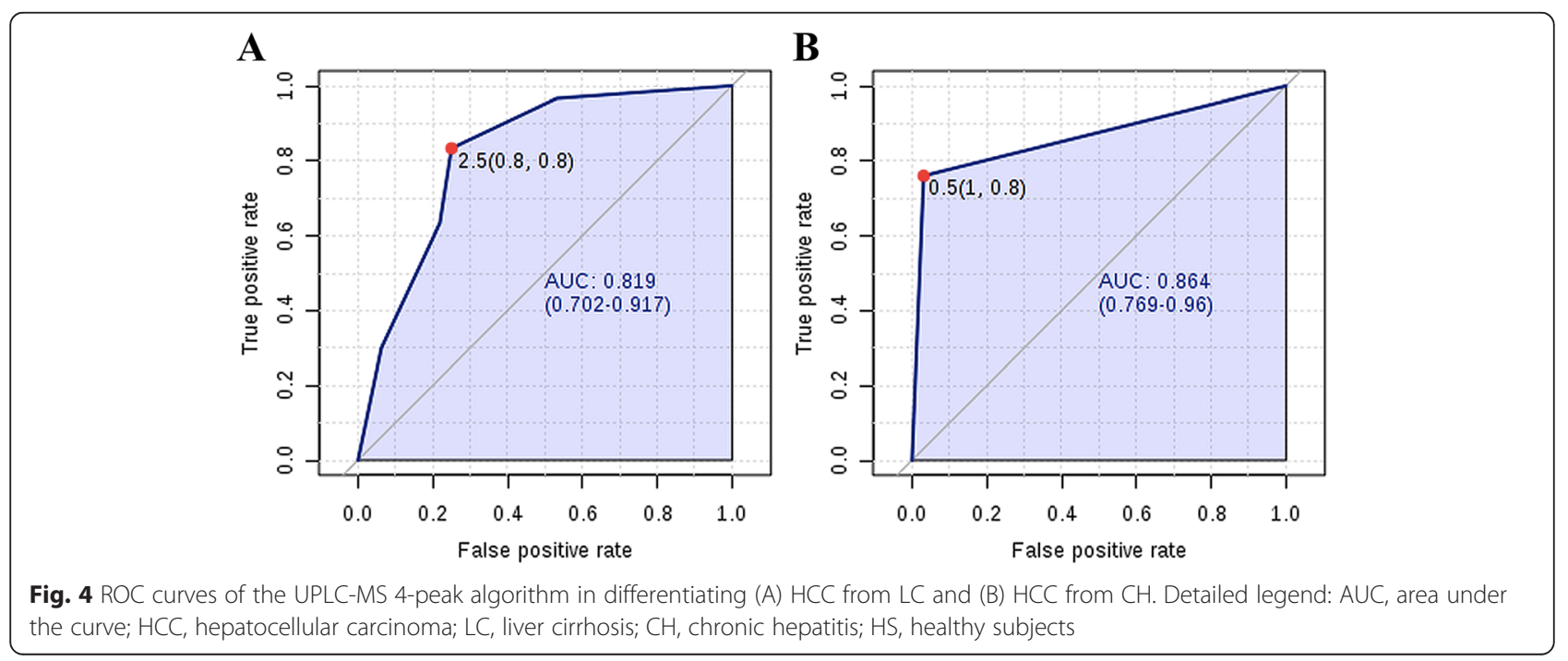


Table 3 Sensitivity and specificity of UPLC-MS profiles, AFP and individual peaks for HCC diagnosis

\begin{tabular}{lccc}
\hline Test & Sensitivity (\%) & Specificity (\%) & ROC AUC \\
\hline HCC versus LC & & & \\
4-ion UPLC-MS model & 78.1 & 63.6 & $0.819^{\mathrm{b}, \mathrm{c}}$ \\
AFP $_{20} \mathrm{ng} / \mathrm{mL}$ & 37.5 & 93.3 & $0.779^{\mathrm{d}}$ \\
AFP $_{200} \mathrm{ng} / \mathrm{mL}$ & 18.8 & 100.0 & $0.779^{\mathrm{d}}$ \\
4-ion UPLC-MS model & 75.0 & 83.3 & 0.856 \\
$\quad$ + AFP $20 \mathrm{ng} / \mathrm{mL}{ }^{\mathrm{a}}$ & & & \\
HCC versus CHB & & & \\
4-ion UPLC-MS model & 93.8 & 80.0 & $0.864^{\mathrm{e}, \mathrm{f}}$ \\
2-ion UPLC-MS model & 96.9 & 80.0 & $0.933^{9}$ \\
RT 3.40_773.5478 n & 100.0 & 100.0 & 1.000 \\
RT 1.87_534.3902 m/z & 100.0 & 100.0 & 1.000 \\
RT 6.25_369.3538 m/z & 100.0 & 100.0 & 1.000 \\
RT 3.45_822.5670 m/z & 100.0 & 100.0 & 1.000 \\
RT 3.59_770.5691 m/z & 100.0 & 100.0 & 1.000 \\
RT 4.23_851.6090 m/z & 100.0 & 100.0 & 1.000 \\
RT 3.99_826.5920 m/z & 100.0 & 100.0 & 1.000 \\
HCC versus HS & & & \\
4-ion UPLC-MS model & 90.6 & 88.2 & 0.946
\end{tabular}

$R O C$ receiver operating characteristic, $A U C$ area under the curve, $H C C$ hepatocellular carcinoma, LC liver cirrhosis, AFP alpha-fetoprotein, UPLC ultra performance liquid chromatography, MS mass spectrometry, CHB chronic hepatitis $B, R T$ retention time, $n$ neutral, $\mathrm{m} / \mathrm{z}$ mass to charge ratio, $H S$ healthy subjects.

${ }^{\text {a }}$ Combination of the 4-ion UPLC-MS model and AFP $20 \mathrm{ng} / \mathrm{mL}$

${ }^{\mathrm{b} C}$ Compared with $\mathrm{AFP}_{20} \mathrm{ng} / \mathrm{mL}$ or $\mathrm{AFP}_{200 \mathrm{ng} / \mathrm{mL}} P=0.616$.

${ }^{\circ}$ Compared with 4-ion UPLC-MS model $+\mathrm{AFP}_{20} \mathrm{ng} / \mathrm{mL}, P=0.610$

${ }^{\mathrm{d} C o m p a r e d}$ with 4-ion UPLC-MS model $+\mathrm{AFP}_{20} \mathrm{ng} / \mathrm{mL}, P=0.312$.

'Compared with 2-ion UPLC-MS model, $P=0.241$.

${ }^{f}$ Compared with individual ions, $P=0.047$.

${ }^{9}$ Compared with individual ions, $P=0.005$

biomarker detection (AFP). Our results also show that the UPLC-MS lipidomic fingerprinting discriminated serum lipidomic expression patterns among patients with HBVHCC, HBV-LC, and CHB.

Studies on lipidomic profiling of HCC are still scarce. Moreover, key data are lacking in the few published studies, such as comprehensive description and assessment regarding patient and background liver disease characterization, group allocation and controls adequacy, and proper performance assessment of the diagnostic model, among others [14].

The results presented herein are innovative, as this study performs a robust evaluation of patients enrolled in a well-established HCC surveillance program. These patients are, therefore, well characterized as to their clinical and laboratory parameters, which ensures the adequacy of the study groups and controls, and allows an unbiased interpretation of the proposed biomarkers and their intra-group level variations.
Table 4 Tentative identification of potential UPLC-MS biomarkers for HCC

\begin{tabular}{|c|c|c|c|}
\hline RT_m/z & Adduct & Identified result & Main class \\
\hline $1.30 \_498.8315$ & {$[\mathrm{M}+\mathrm{H}]+$} & unknown & - \\
\hline $1.32 \_497.5731$ & {$[\mathrm{M}+\mathrm{H}]+$} & unknown & - \\
\hline 1.30_496.6721 & {$[\mathrm{M}+\mathrm{H}]+$} & unknown & - \\
\hline 4.26_540.4255 & {$[\mathrm{M}+\mathrm{H}]+$} & unknown & - \\
\hline 3.40_773.5478 & {$[\mathrm{M}+\mathrm{H}]+$} & $\operatorname{PS}(0-16: 0 / 20: 2)^{a}$ & Glycerophosphoserines \\
\hline 1.87_534.3902 & {$[\mathrm{M}+\mathrm{H}]+$} & unknown & - \\
\hline $6.25 \_369.3538$ & {$[\mathrm{M}+\mathrm{H}]+$} & unknown & - \\
\hline 3.45_822.5670 & {$[\mathrm{M}+\mathrm{H}]+$} & $\mathrm{PS}(\mathrm{O}-18: 0 / 22: 6)^{\mathrm{b}}$ & Glycerophosphoserines \\
\hline 3.59_770.5691 & {$[\mathrm{M}+\mathrm{H}]+$} & $P C(15: 0 / 20: 3)^{c}$ & Glycerophosphocholines \\
\hline 4.23_851.6090 & {$[\mathrm{M}+\mathrm{H}]+$} & $\mathrm{PI}(\mathrm{O}-16: 0 / 20: 1)^{d}$ & Glycerophosphoinositols \\
\hline 3.99_826.5920 & {$[\mathrm{M}+\mathrm{H}]+$} & $P S(O-18: 0 / 22: 4)^{e}$ & Glycerophosphoserines \\
\hline
\end{tabular}

When used as a diagnostic biomarker, AFP is expected to misdiagnose up to $40 \%$ of $\mathrm{HCC}$ cases with a $20 \mathrm{ng} / \mathrm{mL}$ cutoff value [4]. In this study, however, while the UPLCMS-based 4-peak model accurately diagnosed 25 of 32 HCC cases from LC patients, AFP performed poorly, detecting only 12 of 32 cases with a sensitivity of $37.5 \%$ and $93.3 \%$ specificity.

When applied to differentiate HCC in the early stages, the UPLC-MS signature detected very early or early stage HCC with $73.7 \%$ sensitivity and $63.3 \%$ specificity. These data show the potential applicability of UPLC-MS for screening biomarkers for early diagnosis of HCC.

Patients at high risk of HCC development should be screened semi annually using ultrasonography (US). It is known, however, that in most cases US has only acceptable diagnosis accuracy with sensitivity ranging from 58 to $89 \%$ and specificity greater than $90 \%[4,15,16]$. Furthermore, US effectiveness for detecting early-stage HCC is even lower, with a sensitivity of only $63 \%$ [17]. The accuracy of the proposed UPLC-MS 4-peak model for HCC screening and the actual gain in the detection rate need to be further evaluated on larger studies. Nonetheless, the use of this model might improve HCC surveillance and diagnosis, especially in resource-limited regions where patients may have difficult access to US and higher resolution imaging techniques such as $\mathrm{CT}$ scan and magnetic resonance. A lipidomic biomarker and/or profile could be, in turn, detected through a simple, inexpensive and widely accessible enzyme immunoassay or chemiluminescence assay, which would represent a significant reduction on HCC screening costs.

HBV infection can lead to HCC in the absence of cirrhosis. Although little is known about the clinical and epidemiological aspects of HCC in Brazil [18], data from other regions show that 20 to $30 \%$ of patients with 
HBV-related HCC do not present with LC [19]. In this study the rate of $\mathrm{HCC}$ in the absence of cirrhosis was $12.5 \%$. The UPLC-MS 4-peak detected HCC from CHB patients with of $93.8 \%$ sensitivity and a specificity of $80.0 \%$. Furthermore, it was observed that some peaks not included in the first model could differentiate HCC and CHB with $100 \%$ sensitivity and specificity.

We performed a tentative and preliminary identification of the differentially expressed peaks. At this point we have identified 3 glycerophosphoserines, 1 glycerophosphocholine and 1 glycerophosphoinositol, all in significantly lower levels in HCC patients.

Previous studies also have shown lower levels of glycerophosphocolines in HCC patients, which are the most abundant phospholipid in mammalian cellular membranes [11]. This under expression may result from the inflammatory response and consequent higher consumption of these lipids [20, 21]. CHB infection has been associated with alterations in lipid metabolism and a recent study showed that HBV infection altered the metabolic gene expression in a human liver-chimeric mouse model by altering bile acid and cholesterol metabolism as a consequence of impaired bile acid uptake [22].

\section{Conclusions}

Our findings suggest that UPLC-MS lipidomic fingerprinting may be a powerful tool for the identification of diagnostic biomarkers and models for hepatitis B virusrelated HCC. These data showed that the lipid fingerprinting in HCC patients selected a number of lipids that should be functionally investigated to elucidate the pathogenesis of the disease. This technique and the selected peaks show a great potential to improve HCC surveillance in patients with $\mathrm{LC}$ and $\mathrm{CHB}$.

\section{Additional files}

\section{Additional file 1: PCA scores plot based on the UPLC-MS profiling} data for the studied groups. The score plots show the first, second and third principal components. Each dot in the plot represents a patient according to its group. HCC, hepatocellular carcinoma; LC, liver cirrhosis; $\mathrm{CH}$, chronic hepatitis; HS, healthy subjects. (TIF $1120 \mathrm{~kb}$ )

Additional file 2: Distribution of HCC patients according to BCLC staging system. (TIF $62 \mathrm{~kb}$ )

Additional file 3: ROC curves and intensities of the differential ions in the UPLC-MS 4-peak model by RT and $m / z$. ROC curves and intensities of the differential ions in HCC (red boxes) and $\mathrm{CH}$ (green boxes) for (A) RT 1.30_498.8315 m/z; (B) RT 1.32_497.5731 m/z; (C) RT $1.30 \_496.6721 \mathrm{~m} / \mathrm{z}$; (D) RT 4.26_540.4255 m/z. AUC, area under the curve; HCC, hepatocellular carcinoma; LC, liver cirrhosis; $\mathrm{CH}$, chronic hepatitis; $\mathrm{HS}$, healthy subjects. (TIF $4244 \mathrm{~kb}$ )

Additional file 4: ROC curves and intensities of the differential ions by RT and $m / z$. ROC curves and intensities of the differential ions in HCC (red boxes) and CH (green boxes) for (A) RT 3.40_773.5478n; (B) RT 4.23_851.6090 m/z; (C) RT 3.59_770.5691 m/z; (D) RT 3.45_822.5670 m/z; (E) RT 6.25_369.3538 m/z; (F) RT 1.87_534.3902 m/zi (G) RT 3.99_826.5920 m/z.
AUC, area under the curve; HCC, hepatocellular carcinoma; LC, liver cirrhosis; CH, chronic hepatitis; HS, healthy subjects. (TIF 7531 kb)

\section{Competing interests}

The authors declare that they have no competing interests.

\section{Authors' contributions}

AMPC, VMC, KHMC, JRRP, FJC and CFHG were responsible for study conception and design. AMPC, LC, ALC, MSGG, FM and ACSSN recruited the patients, collected the blood samples and clinical and laboratory data and performed data analysis and interpretation. AMPC, VMC and KHMC performed mass-spectrometry analysis and interpretation of data. AMPC performed the statistical analysis and drafted the manuscript. All authors participated in the critical revision, read and approved the final manuscript.

\section{Author's information}

AMPC is currently at her final semester as a PhD Student at the Federal University of Sao Paulo. She has been awarded a highly competitive merit PhD scholarship from Fundação de Amparo à Pesquisa do Estado de São Paulo and two awards for best oral presentation at the Brazilian Congress of Virology. Moreover, the preliminary results of her PhD study have been awarded twice during international conferences. First she received a Student Travel Stipend for oral presentation by the Human Proteome Organisation for the HUPO $11^{\text {th }}$ Annual World Congress and second, she received a Full Young Investigator Bursary for oral presentation of high quality abstract by the European Association for the Study of the Liver for oral presentation at the EASL $50^{\text {th }}$ The International Liver Congress. Furthermore, during the short six years since she graduated as a Pharmacist and Biochemist, she has published 15 scientific articles which have been cited a total of 58 times in SCOPUS.

\section{Acknowledgements}

The Fleury SA Group supported this work and AMPC received a doctorate scholarship from Fundação de Amparo à Pesquisa do Estado de São Paulo FAPESP (no. 2013/03701-0). The funding agencies did not interfere in the scientific aspects of the study.

\section{Author details}

${ }^{1}$ Division of Infectious Diseases, Federal University of Sao Paulo, 781 Pedro de Toledo Street, 15th floor, Sao Paulo, SP 04039032, Brazil. ${ }^{2}$ Fleury Group, Sao Paulo, SP, Brazil. ${ }^{3}$ Department of Gastroenterology, Sao Paulo Clinicas Liver Cancer Group, Instituto do Câncer do Estado de São Paulo, Hospital das Clínicas, Sao Paulo, SP, Brazil. ${ }^{4}$ Department of Infectious Diseases, University of São Paulo School of Medicine, Sao Paulo, SP, Brazil.

Received: 3 July 2015 Accepted: 10 December 2015

Published online: 18 December 2015

\section{References}

1. Ott JJ, Stevens GA, Groeger J, Wiersma ST. Global epidemiology of hepatitis $B$ virus infection: new estimates of age-specific HBsAg seroprevalence and endemicity. Vaccine. 2012;30:2212-9.

2. Ferlay J, Shin HR, Bray F, Forman D, Mathers C, Parkin DM. Estimates of worldwide burden of cancer in 2008: GLOBOCAN 2008. Int J Cancer. 2010; 127:2893-917.

3. Carrilho FJ, Kikuchi L, Branco F, Gonçalves CS, Mattos AA, Brazilian HCC Study Group. Clinical and epidemiological aspects of hepatocellular carcinoma in Brazil. Clinics (Sao Paulo). 2010;65:1285-90.

4. European Association For The Study Of The Liver and European Organisation For Research And Treatment Of Cancer. EASL-EORTC clinical practice guidelines: management of hepatocellular carcinoma. J Hepatol. 2012;56:908-43

5. McGlynn KA, Petrick JL, London WT. Global Epidemiology of Hepatocellular Carcinoma: An Emphasis on Demographic and Regional Variability. Clin Liver Dis. 2015;19:223-38.

6. Bruix J, Sherman M. AASLD Practice Guideline. Management of hepatocellular carcinoma: an update. Hepatology. 2011;53:1020-2.

7. Wenk MR. The emerging field of lipidomics. Nat Rev Drug Discov. 2005;4:594-610.

8. Hu C, van der Heijden R, Wang M, van der Greef J, Hankemeier T, Xu G. Analytical strategies in lipidomics and applications in disease biomarker discovery. J Chromatogr B Analyt Technol Biomed Life Sci. 2009;877:2836-46. 
9. Diamond DL, Syder AJ, Jacobs JM, Sorensen CM, Walters KA, Proll SC, et al. Temporal Proteome and Lipidome Profiles Reveal Hepatitis C Virus-Associated Reprogramming of Hepatocellular Metabolism and Bioenergetics. PLoS Pathog. 2010;6:e1000719. doi:10.1371/journal.ppat.1000719.

10. Patterson AD, Maurhofer $\mathrm{O}$, Beyoglu D, Lanz C, Krausz KW, Pabst T, et al. Aberrant Lipid Metabolism in Hepatocellular Carcinoma Revealed by Plasma Metabolomics and Lipid Profiling. Cancer Res. 2011;71:6590-600

11. Wang B, Chen D, Chen Y, Hu Z, Cao M, Xie Q, et al. Metabonomic profiles discriminate hepatocellular carcinoma from liver cirrhosis by ultraperformance liquid chromatography-mass spectrometry. J Proteome Res. 2012;11:1217-27.

12. Zhou L, Wang $\mathrm{Q}$, Yin $\mathrm{P}$, Xing W, Wu Z, Chen S, et al. Serum metabolomics reveals the deregulation of fatty acids metabolism in hepatocellular carcinoma and chronic liver diseases. Anal Bioanal Chem. 2012;403:203-13.

13. Bligh EG, Dyer WJ. A rapid method of total lipid extraction and purification. Can J Biochem Physiol. 1959;37:911-7.

14. Kimhofer T, Fye H, Taylor-Robinson S, Thursz M, Holmes E. Proteomic and metabonomic biomarkers for hepatocellular carcinoma: a comprehensive review. Br J Cancer. 2015;112:1141-56.

15. Kim CK, Lim JH, Lee WJ. Detection of hepatocellular carcinomas and dysplastic nodules in cirrhotic liver: accuracy of ultrasonography in transplant patients. J Ultrasound Med. 2001;20:99-104.

16. Bolondi L. Screening for hepatocellular carcinoma in cirrhosis. J Hepatol. 2003:39:1076-84

17. Singal A, Volk ML, Waljee A, Salgia R, Higgins $P$, Rogers MA, et al. Meta-analysis: surveillance with ultrasound for early-stage hepatocellular carcinoma in patients with cirrhosis. Aliment Pharmacol Ther. 2009;30:37-47.

18. Kikuchi L, Chagas AL, Alencar RS, Paranaguá-Vezozzo DC, Carrilho FJ. Clinical and epidemiological aspects of hepatocellular carcinoma in Brazil. Antivir Ther. 2013;18:445-9.

19. El-Serag HB. Hepatocellular carcinoma. N Engl J Med. 2011;365:1118-27.

20. Sullentrop F, Moka D, Neubauer S, Haupt G, Engelmann U, Hahn J, et al. 31P NMR spectroscopy of blood plasma: determination and quantification of phospholipid classes in patients with renal cell carcinoma. NMR Biomed. 2002;15:60-8.

21. Lewen Jia JC, Yin P, Lu X, Guowang X. Serum metabonomics study of chronic renal failure by ultra performance liquid chromatography coupled with Q-TOF mass spectrometry. Metabolomics. 2008;4:183-9.

22. Oehler N, Volz T, Bhadra OD, Kah J, Allweiss L, Giersch K, et al. Binding of hepatitis $B$ virus to its cellular receptor alters the expression profile of genes of bile acid metabolism. Hepatology. 2014;60:1483-93.

\section{Submit your next manuscript to BioMed Central and we will help you at every step:}

- We accept pre-submission inquiries

- Our selector tool helps you to find the most relevant journal

- We provide round the clock customer support

- Convenient online submission

- Thorough peer review

- Inclusion in PubMed and all major indexing services

- Maximum visibility for your research

Submit your manuscript at www.biomedcentral.com/submit

C) Biomed Central 